\title{
Balloon-expandable stent angioplasty in the treatment of vertebral artery stenosis in the V2 segment
}

\author{
Xueli Cai ${ }^{1}$, Yixin Wei ${ }^{1}$, Shaojun Ren ${ }^{2}$, Zhiping $\mathrm{Wu}^{1}$, Xiao Peng ${ }^{1}$, Yuejin Huang ${ }^{1}$, Liangtong Huang ${ }^{1}$, Xiumei Liu ${ }^{1}$, \\ Zhihua Yang ${ }^{3}$ \\ ${ }^{1}$ Department of Neurology, Lishui Hospital of Zhejiang University (the Central Hospital of Lishui), Zhejiang, China \\ ${ }^{2}$ Department of Orthopaedics, Lishui Hospital of Zhejiang University (the Central Hospital of Lishui), Zhejiang, China \\ ${ }^{3}$ Department of Neurology, the First Affiliated Hospital of Guangzhou Medical University, Guangzhou, Guangdong, China
}

\begin{abstract}
Introduction: Vertebral artery stenosis is a major cause of posterior circulation ischemia in the elderly. There is not a clear consensus on the optimal therapeutic approach for symptomatic extracranial vertebral artery stenosis.

Aim: To evaluate the feasibility and efficacy of balloon-expandable stent angioplasty in the treatment of vertebral artery stenosis in the V2 segment.

Material and methods: Five patients with vertebral artery stenosis (V2 segment) and treatment of percutaneous transluminal stenting from July 2009 to June 2014 were retrospectively evaluated. All patients underwent color Doppler, transcranial color Doppler (TCD), CT angiography (CTA) and cerebral digital subtraction angiography (DSA) preoperatively. Whether there was osseous oppression was determined according to neck computed tomography (CT) and CTA. After the surgery, angiography was performed to determine if there was infarction or bleeding in the intracranial vertebral artery, basilar artery and posterior cerebral artery. The surgical parameters, residual stenosis, complications, etc. were recorded and evaluated. The patients were followed up accordingly.

Results: Five patients ( 3 males, 2 females; average age of $66 \pm 4.2$, range of 54-75) were enrolled in the study. Balloon-expandable stents were successfully implanted in the 5 patients. The mean residual stenosis after the balloon-expandable stenting (preoperative: average, $87.0 \pm 6.6 \%$, range: $75-93 \%$ ) was $12.6 \pm 7.8 \%$ (range: $5-25 \%)$. The clinical symptoms disappeared or receded. No serious complications occurred.

Conclusions: The balloon-expandable stent angioplasty seemed to be feasible and efficacious in treating vertebral artery stenosis in the V2 segment. Further study with a large sample size is needed.
\end{abstract}

Key words: posterior circulation ischemia, stent angioplasty, restenosis, vertebrobasilar insufficiency.

\section{Introduction}

Vertebral artery stenosis is a major cause of posterior circulation ischemia in the elderly [1]. The stroke incidence in patients with vertebral artery stenosis can be as high as $10.9 \%$ [2]. Symptomatic vertebrobasilar artery stenosis usually has poor prognosis, with the death and permanent morbidity rate of $10 \%$ as a result of ischemic stroke even after positive drug treatment [1]. There is not a clear consensus on the optimal therapeutic approach for symptomatic extracranial vertebral artery stenosis. The treatment methods for vertebrobasilar artery stenosis include conservative management and surgical management. However, neither of these methods is efficacious. Conservative management

\section{Address for correspondence}

Zhihua Yang, Department of Neurology, the First Affiliated Hospital of Guangzhou Medical University, 151 Yanjiang Road, Guangzhou, 510120 Guangdong, China, phone: +86 02083062114 , e-mail: yangzhihuazh@163.com 
typically includes modification of systemic arterial hypertension, glucose intolerance, serum lipid profile and treatment with antithrombotic agents [3]. Surgical management of symptomatic vertebral disease includes subclavian vertebral roof-patch angioplasty, reimplantation of the vertebral origin, carotid-vertebral vein bypass and vertebral ostial endarterectomy [4]. Artery bypass surgery is a difficult operation with considerable trauma and high incidence of complications. It is reported to have a mortality rate of $30 \%$ perioperatively [5].

The vertebral artery can be divided into four segments. The V1 segment arising from the subclavian artery enters the transverse foramen of the sixth cervical vertebra. The V2 segment passes through the foramina of the C1-C6 transverse process [6]. With the development of endovascular stents and neurointervention, stent angioplasty is increasingly used in the treatment of vertebral artery stenosis in the proximal segment (V1 segment) and intracranial segment (segments V3 and V4) [7]. However, there has been no previous report regarding endovascular treatment in the V2 segment of vertebral artery stenosis. It was traditionally considered that endovascular stenting was not appropriate for the V2 segment of vertebral artery stenosis in fear of vertebral artery stenosis caused by cervical bone hyperplasia [8]. Recently, a consensus among Chinese experts has reported that cervical bone hyperplasia is not the main cause of posterior circulation ischemia [9]. There is no significant difference in the degree of cervical bone hyperplasia between the elderly with posterior circulation ischemia and the elderly without posterior circulation ischemia. It is the vascular risk factors that contribute to posterior circulation ischemia [10, 11]. Continuous dynamic vertebral angiography showed that there were only very few individuals with vertebral artery compression caused by osteophytes. Doppler ultrasound examination showed no statistically significant difference in compression rate of the extracranial vertebral artery between patients with and without posterior circulation symptoms [12].

Endovascular treatment might be an alternative method for patients failing to respond to conservative treatment. Compared with stent angioplasty in the V1 and V3 segment, it needs careful assessment for the bony structure through neck computed tomography (CT) and CT angiography (CTA) in the treatment of the V2 segment. It is not suitable for endovascular treatment if bony structure oppression exists.

\section{Aim}

In the present study, we retrospectively reviewed 5 cases with arterial stenosis in the V2 segment and posterior circulation ischemia, who were treated with balloon-expandable stent angioplasty. We aimed to evaluate the feasibility and efficacy of balloon-expandable stent angioplasty in the treatment of vertebral artery stenosis in the V2 segment. The main endpoints were residual stenosis, improvement of symptoms and complication occurrence.

\section{Material and methods}

\section{Patients}

This study was approved by the ethic committee of our hospital and signed informed consent was obtained from each patient. We performed a retrospective evaluation on demographic, clinical and radiographic data that were collected on 5 patients with vertebral artery stenosis (V2 segment) and treatment of percutaneous transluminal stenting from July 2009 to June 2014. The disease was diagnosed by cerebral angiography. The clinical symptoms were dizziness, vertigo and unsteady gait. The inclusion criteria were the patient's failure to respond to medicinal treatment for above half a year and the patient lacking osseous oppression in the stenotic area according to CT and CTA. Cases of cerebral infarction within 4 weeks were excluded.

\section{Imaging methods}

All patients underwent color Doppler, transcranial color Doppler (TCD), CTA and cerebral digital subtraction angiography (DSA) preoperatively (Photo $1 \mathrm{~A}, \mathrm{~B})$. The incidence of vertebrobasilar artery stenosis was determined according to the North American Symptomatic Carotid Endarterectomy Trial (NASCET) for measuring the rate of internal carotid stenosis [13]. Whether there was osseous oppression was determined according to neck CT and CTA.

\section{Surgical procedures}

Conventional electrocardiography (ECG), hemostatic function, head magnetic resonance imaging (MRI), ultrasound of neck blood vessel, CTA, aortic arch and cerebral CTA were performed. The following were assessed: general physical condition, nervous system, stenosed blood vessel, stenosis, the type of 

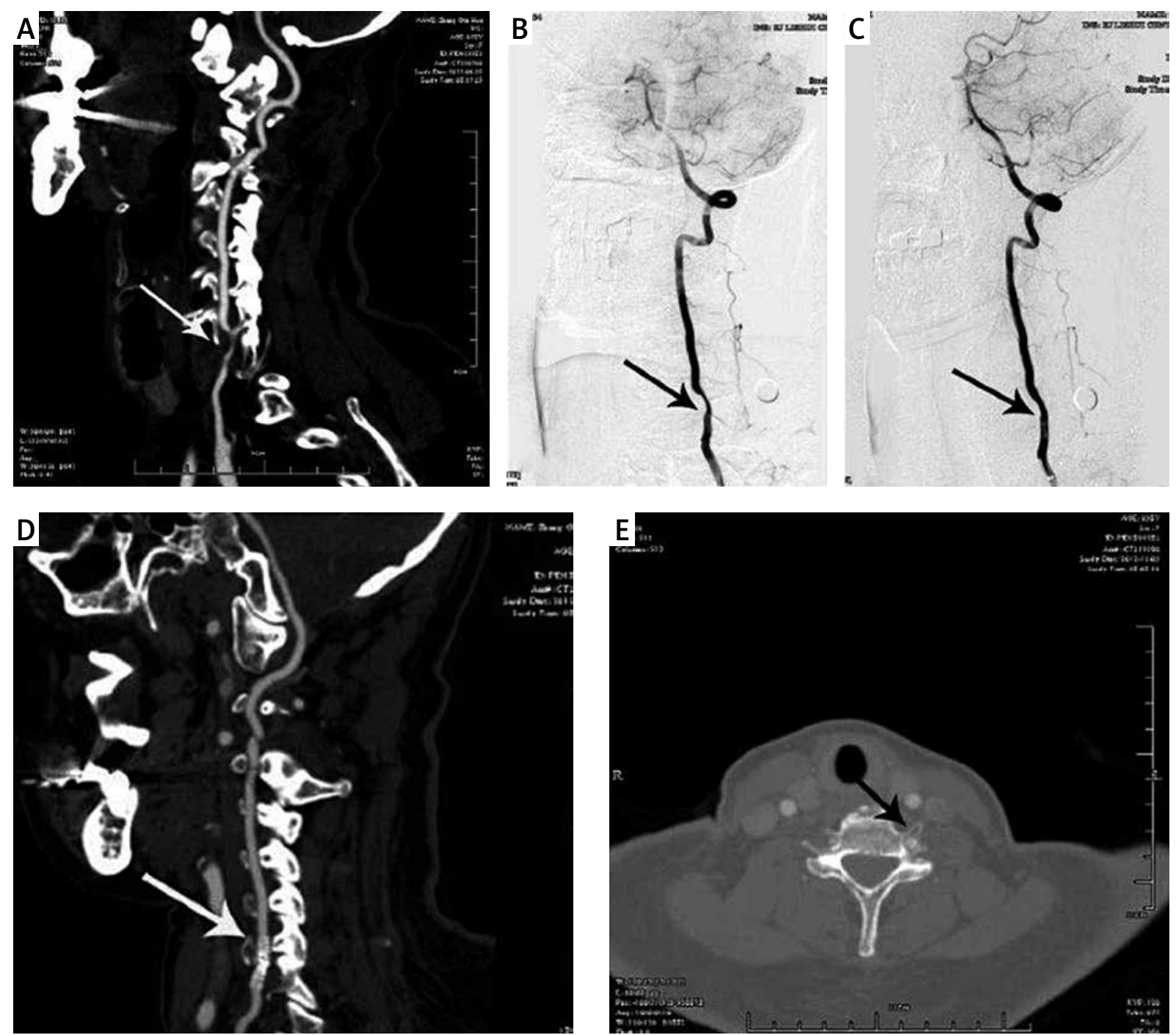

Photo 1. The preoperative and postoperative CTA and DSA of a patient with vertebral artery stenosis. A, B - The preoperative CTA (A) and DSA (B) showed stenosis in V2 segment (as the arrow shows). C - Postoperative DSA showed that the surgery was successful and the stenosis disappeared (as the arrow shows). D, E-CTA (D) and DSA (E) 1 year postoperatively showed no restenosis in the vertebral artery

plaque, dominant vertebral artery and posterior inferior cerebellar artery.

The patient received oral administration of Aspirin (Bayer, Leverkusen, Germany) of 300 mg/day and clopidogrel (Plavix, Sanofi-Aventis France) of $75 \mathrm{mg} /$ day for three days before the procedure. Nimodipine was administered intravenously $2 \mathrm{~h}$ before the procedure. The dosage was regulated according to the blood pressure. The surgery was performed under local anaesthesia.

The right femoral artery was punctured with the Seldinger method [14] and a 6-8 Fr sheath was placed. Heparin of $0.75 \mathrm{mg} / \mathrm{kg}$ was injected intrave- nously for systemic heparinization. The Envoy/MPD 6-8 F guiding catheter (Cordis Corporation, Miami Lakes, USA) was placed in the ipsilateral subclavian artery with the guidance of a super smooth guide wire (Terumo Corporation, Tokyo, Japan). Multi-angle (positive, lateral and oblique position) magnification angiography clearly showed the stenosed section of the vertebral artery. After evaluation of the stenosis rate, the proper size of ball stents (3-4 mm × 12-18 mm, Apoll 4008, MicroPort, Z. JHigh-Tech Park Shanghai, China) were chosen according to the normal lumen of the distal part of the stenosis section. After being properly remolded, 
the 0.014" wire was transported from the vertebral artery stenosis site to the vertebral artery distal through the guiding catheter under the guidance of the "road map" of DSA. The ball stent was placed across the stenosis with the guidance of the wire. The expansion pressure gradually increased to 6-12 atm for 5-8 s. A quick initial expansion speed may easily result in stent shifting, while too low expansion may lead to a relatively long time of ischemia and complications. If there was pain locally or in the upper limb, further expansion was not allowed. Then angiography was performed to assess the stenting and the balloon was withdrawn. Angiography was performed to determine whether there was infarction or bleeding in the intracranial vertebral artery, basilar artery and posterior cerebral artery [15].

The artery sheath was retained postoperatively and was removed when heparin neutralized naturally (about $4 \mathrm{~h}$ after the surgery). Heart rate and blood pressure were continuously monitored by ECG. The blood pressure was controlled and kept 10-20 $\mathrm{mm} \mathrm{Hg}$ lower than the preoperative blood pressure, unless there were untreated stenosed blood vessels. Low molecular heparin was injected subcutaneously every $12 \mathrm{~h}$ for 3 consecutive days to prevent postoperative thrombosis. The patient followed the regime of oral administration of Aspirin of $300 \mathrm{mg} /$ day and clopidogrel of $75 \mathrm{mg} /$ day for 6 months. Aspirin of $100 \mathrm{mg} /$ day could be administered for an appropriate period depending on the patient's situation.

\section{Follow up}

The vertebral artery and subclavian artery were examined by color Doppler ultrasound and CTA
1 week, 1 month, 3 months and 6 months after the surgery.

\section{Results}

Five patients ( 3 males, 2 females; average age: $66 \pm 4.2$ years, range: $54-75$ years) were enrolled in the study. The baseline data are shown in Table I.

Five balloon-expandable stents were successfully implanted in the five patients without any operational complications (Photo $1 \mathrm{C}$ ). The average surgical time was $1.7 \pm 0.25 \mathrm{~h}$ (range: $1.5-2 \mathrm{~h}$ ). The mean residual stenosis after the balloon-expandable stenting (preoperative: $87.0 \pm 6.6 \%$ on average, range: $75-93 \%$ ) was $12.6 \pm 7.8 \%$ (range: $5-25 \%$ ). The clinical symptoms disappeared or receded.

The color Doppler ultrasound and CTA showed that there was no restenosis in the vertebral artery or subclavian artery during the follow-up of 6-12 months (Photo $1 \mathrm{D}, \mathrm{E}$ ).

\section{Discussion}

The 5 patients with arterial stenosis in the V2 segment and posterior circulation ischemia were treated with balloon-expandable stent angioplasty successfully. No complications were observed after a follow-up of 6-12 months. The method proved effective in the present study.

Regarding the complicated condition in the V2 segment, neck CT and CTA have to be carefully performed and assessed to determine if there exists bony structure-caused compression. In the present study, all the 5 patients failed to respond to the medicinal treatment of over half a year, and none

Table I. Baseline data of patients

\begin{tabular}{|c|c|c|c|c|c|c|c|c|c|}
\hline Case & Age & Sex & $\begin{array}{c}\text { Site } \\
\text { of lesion }\end{array}$ & Symptoms & Comorbidity & $\begin{array}{l}\text { Surgical } \\
\text { time }[\mathrm{h}]\end{array}$ & $\begin{array}{c}\text { SR } \\
\text { pre. (\%) }\end{array}$ & $\begin{array}{c}\text { SR } \\
\text { post. (\%) }\end{array}$ & $\begin{array}{c}\text { Angioplasty } \\
\text { [mm] }\end{array}$ \\
\hline 1 & 57 & $\mathrm{~F}$ & Left & $\begin{array}{c}\text { Vertigo, } \\
\text { hemiparesis }\end{array}$ & $\begin{array}{c}\text { Hypertension, } \\
\text { diabetes }\end{array}$ & 2 & 85 & 10 & 3.0 \\
\hline 2 & 68 & $M$ & Right & $\begin{array}{c}\text { Vertigo, } \\
\text { dysarthria }\end{array}$ & $\begin{array}{c}\text { Hypertension, } \\
\text { diabetes }\end{array}$ & 1.5 & 92 & 5 & 2.5 \\
\hline 3 & 72 & $\mathrm{~F}$ & Right & $\begin{array}{c}\text { Vertigo, } \\
\text { hemiparesis }\end{array}$ & Hypertension & 1.5 & 90 & 25 & 3.0 \\
\hline 4 & 54 & $M$ & Left & $\begin{array}{l}\text { Dizziness, } \\
\text { hemiplegia }\end{array}$ & Hypertension & 1.5 & 93 & 18 & 2.5 \\
\hline 5 & 75 & $M$ & Right & $\begin{array}{c}\text { Vertigo, } \\
\text { dysphagia }\end{array}$ & Hypertension & 2 & 75 & 5 & 3.0 \\
\hline
\end{tabular}

F-female, $M$ - male, SR - stenosis rate, pre. - preoperatively, post. - postoperatively. 
showed bony structure-caused compression according to the imaging.

Intraoperative embolus loss is a serious complication in endovascular stent angioplasty. To date, there still a lack of reports on vertebrobasilar artery stent angioplasty with a large number of cases. It is still controversial whether the embolus protection technique should be used in vertebrobasilar artery stent angioplasty. Because the vertebral arteries are thinner and commonly form an angle with the subclavian arteries, with the protection device there readily occurs traction with the stent, which may cause displacement and deformation of the stent and difficulty in removing the protection device itself, and it is seldom used in vertebrobasilar artery stenosis [16]. In this study, the embolus protection technique was not used and perioperative stroke did not occur.

Vertebral artery restenosis after stent implantation has attracted more attention in recent years [17]. Restenosis can easily occur after stent angioplasty. There are several factors which may promote restenosis, such as the elasticity of the blood vessel, vascular remodeling, thrombosis, excessive proliferation and migration of vascular smooth muscle cells. In addition, excessive proliferation of vascular smooth muscle cell may play a more important role in the occurrence of restenosis. Incomplete stent expansion is an important factor of stent thrombosis after stenting, which increases the incidence of stent thrombosis and restenosis [18]. Thus, the residual stenosis rate should be reduced as far as possible. Multi-angle imaging should be performed to control the residual stenosis rate lower than $20 \%$. Post-balloon dilatation should be performed in patients with residual stenosis greater than $20 \%$. The antiplatelet treatment and the stent type play important roles in restenosis after stent angioplasty [17]. The SSYLVIA study investigators reported that diabetes, preoperative blood vessel with small diameter and postoperative residual stenosis higher than $30 \%$ were high risk factors of restenosis during the first 6 postoperative months [19]. We suggest that strict postoperative management of antiplatelet, diabetes, hypertension and hyperlipidemia might be the basis for the prevention of restenosis. No restenosis occurred in the 5 patients during the follow-up. Long-term clinical observation and imaging review is necessary.

There are several limitations to this study, such as the very small sample size and the retrospective nature. Long-term follow-up data on restenosis are lacking. Nonetheless, the significant and durable improvement in clinical symptoms over the follow-up period indicates an important role for balloon-expandable stent angioplasty for vertebral artery stenosis. Further study with a large sample size is needed.

\section{Conclusions}

The balloon-expandable stent angioplasty seemed to be feasible and efficacious in treating vertebral artery stenosis in the $\mathrm{V} 2$ segment.

\section{Conflict of interest}

The authors declare no conflict of interest.

\section{References}

1. Gondim FA, Cruz-Flores S, Moore J, al-Joundi T. Angioplasty and stenting for symptomatic basilar artery stenosis. J Neuroimaging 2002; 12: 55-8.

2. Qureshi Al, Suri MFK, Ziai WC, et al. Stroke-free survival and its determinants in patients with symptomatic vertebrobasilar stenosis: a multicenter study. Neurosurgery 2003; 52: 1033-40.

3. Maier IL, Karch A, Lipke C, et al. Transluminal angioplasty and stenting versus conservative treatment in patients with symptomatic basilar artery stenosis: perspective for future clinical trials. Clin Neuroradiol 2016; PMID:27506672.

4. Weber W, Mayer TE, Henkes $H$, et al. Stent-angioplasty of intracranial vertebral and basilar artery stenoses in symptomatic patients. Eur J Radiol 2005; 55: 231-6.

5. Spetzler RF, Hadley MN, Martin NA, et al. Vertebrobasilar insufficiency: part 1: microsurgical treatment of extracranial vertebrobasilar disease. J Neurosurg 1987; 66: 648-61.

6. Hauth EA, Gissler HM, Drescher R, et al. Angioplasty or stenting of extra-and intracranial vertebral artery stenoses. Cardiovasc Intervent Radiol 2004; 27: 51-7.

7. Haitao LU. Drug-eluting stent for the treatment of symptomatic vertebral artery stenosis. J Clin Radiol 2012; 17: 488-90.

8. Rozeman AD, Hund $H$, Westein $M$, et al. Duplex ultrasonography for the detection of vertebral artery stenosis: a comparison with CT angiography. Brain Behav 2017; 7: e00750.

9. Li Y. Chinese expert consenus on posterior circulation ischemia. Chin J Inter Med 2006; 45: 786-7.

10. Katsanos AH, Kosmidou M, Kyritsis AP, Giannopoulos S. Is vertebral artery hypoplasia a predisposing factor for posterior circulation cerebral ischemic events? A comprehensive review. Eur Neurol 2013; 70: 78-83.

11. Subramanian G, Silva J, Silver F, et al. Risk factors for posterior compared to anterior ischemic stroke: an observational study of the Registry of the Canadian Stroke Network. Neuroepidemiology 2009; 33: 12-6.

12. Sakaguchi M, Kitagawa K, Hougaku H, et al. Mechanical compression of the extracranial vertebral artery during neck rotation. Neurology 2003; 61: 845-7.

13. Saba L, Mallarini G. A comparison between NASCET and ECST 
methods in the study of carotids: evaluation using multi-detector-row CT angiography. Eur J Radiol 2010; 76: 42-7.

14. Mangar D, Thrush DN, Connell GR, Downs JB. Direct or modified Seldinger guide wire-directed technique for arterial catheter insertion. Anesth Analg 1993; 76: 714-7.

15. Wachowski M, Polguj M, Ścibór J, Majos A. Ischaemia of the medullary cone after stent-graft implantation in a patient with abdominal aortic aneurysm - a case study. Videosurgery Miniinv DOI: https://doi.org/10.5114/wiitm.2017.70326.

16. Li J, Xu Y, Huang Y, et al. Endovascular stenting in the treatment of symptomatic verticular-basilar artery stenosis. J Apoplexy Nervous Dis 2008; 25: 484-6.

17. Yang JW, Jiao LQ, Miao ZR, et al. Vertebral artery origin restenosis after stent implantation: an analysis of risk factors. Chin J Cerebrovasc Dis 2009; 6: 10-4.

18. Lemesle G, Delhaye C, Bonello L, et al. Stent thrombosis in 2008: definition, predictors, prognosis and treatment. Arch Cardiovasc Dis 2008; 101: 769-77.

19. Investigators SS. Stenting of symptomatic atherosclerotic lesions in the vertebral or intracranial arteries (SSYLVIA) study results. Stroke 2004; 35: 1388-92.

Received: 8.12.2017, accepted: 15.01.2018. 